\title{
VP-15
}

\section{Technical aspects in difficult laparoscopic distal splenopancreatectomy}

\author{
Ivelin TAKOROV', Dimitriya VALCHEVA ${ }^{* 1}$, Tsonka LUKANOVA', Dimitar DIMITROV', Mariya ABRASHEVA ${ }^{2}$ \\ ${ }^{1}$ First Clinic of Abdominal Surgery, Military Medical Academy, Sofia, Bulgaria \\ ${ }^{2}$ Anaesthesiology and Intensive Care, Military Medical Academy, Sofia, Bulgaria
}

Introduction: Laparoscopic distal pancreatectomy is the gold standard nowadays. The infiltration of splenic artery near to its origin as well as engagement of splenic vein near to its confluence with superior mesenteric vein could be a reason for technical difficulties. In such cases the most easily accessible major landmark is the common hepatic artery. Transection of the pancreatic neck and splenic vein is essential to reach the dorsal aspect of the splenic artery at the level of the celiac trunk. The aim of this video is to present our approach to retroperitoneal access of the splenic artery.

Methods: We present two cases of laparoscopic distal splenopancreatectomy, one of them for cancer of the pancreatic body, and the other one with pseudocyst of the pancreatic body. Mean age of the two patients was 59.5.

Results: Totally laparoscopic splenopancreatectomy with hepatoduodenal and celiac lymph node dissection was performed. Peripancreatic invasion near to the origin of the splenic artery was observed. Stepwise pancreatic transection at the neck, followed by transection of splenic vein were done in both cases. In the second one major venous bleeding occured and was managed by clipping and division of the splenic vein. The mean operative time was 240 minutes with insignificant blood loss. The patients had uneventful hospital stay and were discharged on the 7.5th postoperative day.

Conclusions: Laparoscopic distal pancreatectomy can be technically difficult and should be performed only by surgeons with sufficient experience in both open pancreatic surgery and advanced laparoscopic gastrointestinal surgery. 